\title{
Needs, Challenges and UK Survivorship Initiative and Solutions
}

Side effects of treatment such as erectile dysfunction, are a significant problem. The difficulties experienced by patients are re-enforced by a report from the Prostate Cancer UK Charity. Of the 10,000 prostate cancer patients who were studied, $80 \%$ had side effects of therapy including erectile dysfunction [1]. For ED it can be up to 50-60\% [1]. Survivors themselves indicated these needs had not been adequately assessed [2]. In conclusion, psychosexual care in particular requires further investigation. This study will therefore add to the body of evidence, in particular, psychosexual support for men who have had surgery for prostate cancer.

The short and long-term impacts of anti-cancer treatments on patients have been recurrent themes in the cancer survivorship literature [3]. Side effects of treatment are a significant problem [3]. These unmet needs are a significant burden on the NHS. The difficulties experienced by patients are re-enforced by a report from the Prostate Cancer UK Charity. Of the 10,000 prostate cancer patients who were studied, $80 \%$ had side effects of therapy including erectile dysfunction and unaddressed medical co-morbidities [1]. Survivors themselves indicated these needs had not been adequately assessed [2]. These unmet needs in particular require further investigation. This study will therefore add to the body of evidence, in particular, psychosexual support for men who have been potentially cured of cancer.

\section{References}

1. Townsend A. Support for men newly diagnosed with prostate cancer. Nurs Stand. 2010;25:40-5.

2. Smith T, Stein KD, Mehta CC, Kaw C, Kepner JL, Buskirk T, Stafford J, Baker F. The rationale, design, and implementation of the American Cancer Society's studies of cancer survivors. Cancer. 2007;109:1-12.

3. Rowland JH, Hewitt M, Ganz PA. Cancer survivorship: a new challenge in delivering quality cancer care. J Clin Oncol. 2006;24:5101-4. 